\title{
CENTRAL AND ALMOST CONSTRAINED SUBSPACES OF BANACH SPACES
}

\begin{abstract}
T. S. S. R. K. RAO
Abstract. In this paper we continue the study of central subspaces initiated in [2] and its infinite version called almost constrained subspaces. We are interested in studying situations where these intersection properties of balls lead to the existence of a linear projection of norm one. We show that every finite dimensional subspace is a central subspace only in Hilbert spaces. By considering direct sums of Banach space we give examples where central subspaces are almost constrained or one-complemented. We show that a $M$-ideal can fail to be a central subspace, answering a question raised in [2].
\end{abstract}

Mathematics subject classification (2010): Primary 46B20, Secondary 41A50,46E15.

Keywords and phrases: Central subspaces, almost constrained subspaces, one-complemented subspaces, Chebychev centres.

\section{REFERENCES}

[1] D. AmIR, Characterizations of inner product spaces, Operator Theory: Advances and Applications, 20. Birkhäuser Verlag, Basel, 1986.

[2] P. Bandyopadhyay and T. S. S. R. K. RaO, Central subspaces of Banach spaces, J. Approx. Theory 103 (2000) 206-222.

[3] P. Bandyopadhyay and S. Dutta, Almost constrained subspaces of Banach spaces, Proc. Amer. Math. Soc. 132 (2004) 107-115.

[4] P. Bandyopadhyay AND S. DutTa, Almost constrained subspaces of Banach spaces. II, Houston J. Math. 35 (2009) 945-957.

[5] M. BARONTI AND P. PAPINI, Norm-one projections onto subspaces of finite codimension in $l_{1}$ and $c_{0}$, Period. Math. Hungar. 22 (1991) 161-174.

[6] M. BARONTI AND P. PAPINI, Bicontractive projections in sequence spaces and a few related kinds of maps, Comment. Math. Univ. Carolin. 30 (1989) 665-673.

[7] J. Blatter And E. W. Cheney, Minimal projections on hyperplanes in sequence spaces, Ann. Mat. Pura Appl. 101 (1974) 215-227.

[8] G. Godefroy, N. J. Kalton And P. D. Saphar, Unconditional ideals in Banach spaces, Studia Math. 104 (1993) 13-59.

[9] P. Harmand, D. Werner And W. Werner, $M$-ideals in Banach spaces and Banach algebras, Lecture Notes in Mathematics, 1547. Springer-Verlag, Berlin, 1993. viii+387 pp.

[10] R. B. Holmes, Geometric functional analysis and its applications, Graduate Texts in Mathematics, No. 24. Springer-Verlag, New York-Heidelberg, 1975. $x+246$ pp

[11] A. KaminsKa, H. J. LeE AND G. LewiCKI, Extreme and smooth points in Lorentz and Marcinkiewicz spaces with applications to contractive projections, Rocky Mountain J. Math. 39 (2009) $1533-1572$.

[12] S. V. Konyagin, A remark on renormings of nonreflexive spaces and the existence of a Chebyshev center (Russian) Vestnik Moskov. Univ. Ser. I Mat. Mekh. 1988, no. 2, 81-82; translation in Moscow Univ. Math. Bull. 43 (1988), no. 2, 55-56.

[13] H. E. LACEY, The isometric theory of classical Banach spaces, Die Grundlehren der mathematischen Wissenschaften, Band 208. Springer-Verlag, New York-Heidelberg, 1974. x+270 pp.

[14] J. Lindenstrauss, Extension of compact operators, Mem. Amer. Math. Soc. No. 48 1964, 112 pp. 
[15] J. Lindenstrauss, On projections wirh norm 1 -an example, Proc. Amer. Math. Soc., 15 (1964) 403-406.

[16] T. S. S. R. K. RaO, On ideals in Banach spaces, Rocky Mountain J. Math. 31 (2001) 595-609.

[17] T. S. S. R. K. RaO, Chebyshev centres and centrable sets, Proc. Amer. Math. Soc. 130 (2002) $2593-$ 2598 (electronic).

[18] Ulf UtTersRud, On M-ideals and the Alfsen-Effros structure topology, Math. Scand. 43 (1978) 369-381 (1979).

[19] L. VeSELÝ, Generalized centers of finite sets in Banach spaces, Acta Math. Univ. Comenian. (N.S.) 66 (1997) 83-115.

[20] L. VESELÝ, Chebyshev centers in hyperplanes of $c_{0}$, Czechoslovak Math. J. 52 (127) (2002) $721-$ 729. 